\title{
Inferior vena cava anomalies: Incidental finding in two cases during computed tomography scan
}

\section{Mubarak MY', Izyan I², Najwa $\mathrm{P}^{3}$}

${ }^{1}$ Mohd Yusof Mubarak, Radiologist, ${ }^{2}$ Ismail Izyan, Medical Officer, Diagnostic Imaging Department, Tengku Ampuan Afzan Hospital, Jalan Tanah Putih, Pahang, Malaysia, ${ }^{3}$ Paiman Najwa, Medical Officer, National Cancer Institute, Putrajaya, Malaysia

\begin{abstract}
Inferior vena cava anomaly is usually asymptomatic. It is rare and commonly detected while performing abdominal imaging for other clinical situations. The anomalies can mimic normal anatomical structures. Thus, the identification of the anomalies is important to avoid complication during abdominal procedures. We report two cases of inferior vena cava anomalies found during Computed Tomography scan of our elective cases.
\end{abstract}

Key words: Cardinal veins, Duplication, Inferior vena cava anomaly, Transposition

\section{INTRODUCTION}

Grom many literature reviews, most cases of inferior - vena cava (IVC) anomalies are diagnosed as a part of incidental finding. Though patient may be asymptomatic, the contrasted Computed Tomography (CT) scan of the abdomen is performed to assess abdominal diseases, which might render the possibility of invasive intervention and surgery. Thus, careful assessment of the $\mathrm{CT}$ images is mandatory as not to miss such anomalies as it may complicate the course of treatment. Being aware about the presence of the IVC anomaly, we are able to assist the clinician in managing the patient and anticipating possible complications.

\section{CASE REPORT}

Case 1: A 42-year old woman underwent Contrast Enhanced Computed Tomography (CECT) scan of the abdomen for abdominal pain. She had altered bowel habit for the past two months but had no constitutional symptoms. The ultrasound and colonoscopy examinations were uneventful. The CECT scan examination revealed no abnormality. However, she was incidentally found to have double inferior vena cava. The left IVC was larger and communicated with the right common iliac vein. The left IVC crossed to the right and united with the suprarenal IVC. The right IVC was

Address for correspondence

Mr. Mohd Yusof Mubarak

Radiologist, Tengku Ampuan Afzan Hospital

Jalan Tanah Putih, Pahang, Malaysia.

E-mail: adibawazif@yahoo.com continuous inferiorly with the right common iliac vein and superiorly united with the suprarenal IVC

Case 2: A 37-year old man was found to have liver mass on ultrasound scan examination. He had right hypochondrium pain and poor appetite for three months. The CECT scan of the abdomen showed nodular liver margin with right lobe liver masses. The diagnosis of multicenteric hepatoma was made. He had portal vein and superior mesenteric vein thrombosis. The suprarenal IVC was on the right however the infra renal IVC was on the left side and received the venous drainage from right and left common iliac veins (Figure 3,4 ).

\section{DISCUSSION}

The development of inferior vena cava (IVC) begins during the six to eight weeks of embryogenesis ${ }^{1}$. It is a very complex process involving the development, regression, anastomosis and replacement of three pairs of cardinal veins. These veins following the sequence of appearance and regression are the posterior cardinal, the subcardinal and the supracardinal. The normal right sided IVC is divided into four parts: a) the infrarenal segment derives from the right supracardinal vein; b) the renal segment develops from the right supracardinal and postsubcardinal anastomosis, c) the hepatic segment from the right hepatic vein, and d) the suprarenal segment from the right subcardinal vein². The IVC anomalies result from the abnormal regression or abnormal persistence of the embryonic veins. 

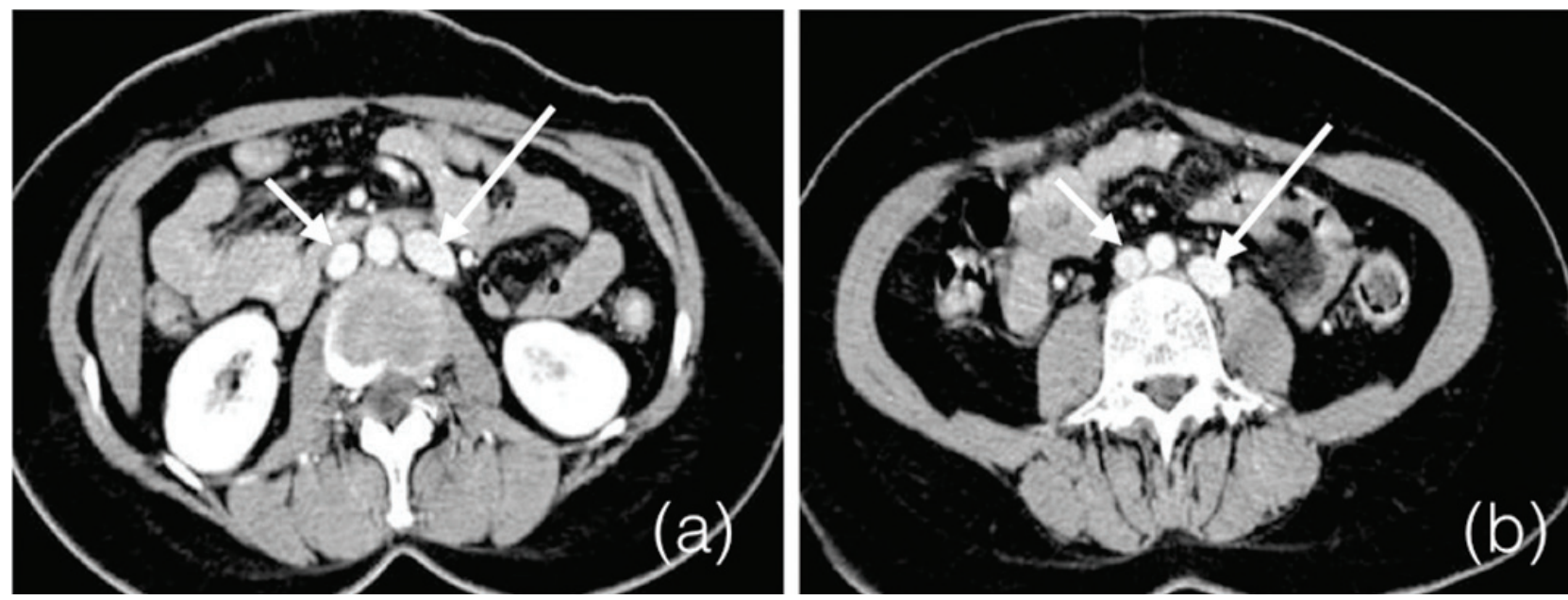

Figure 1: Axial CT images: The right IVC (short arrow) and left IVC (long arrow) are enhancing and clearly visualized in (a) and (b).
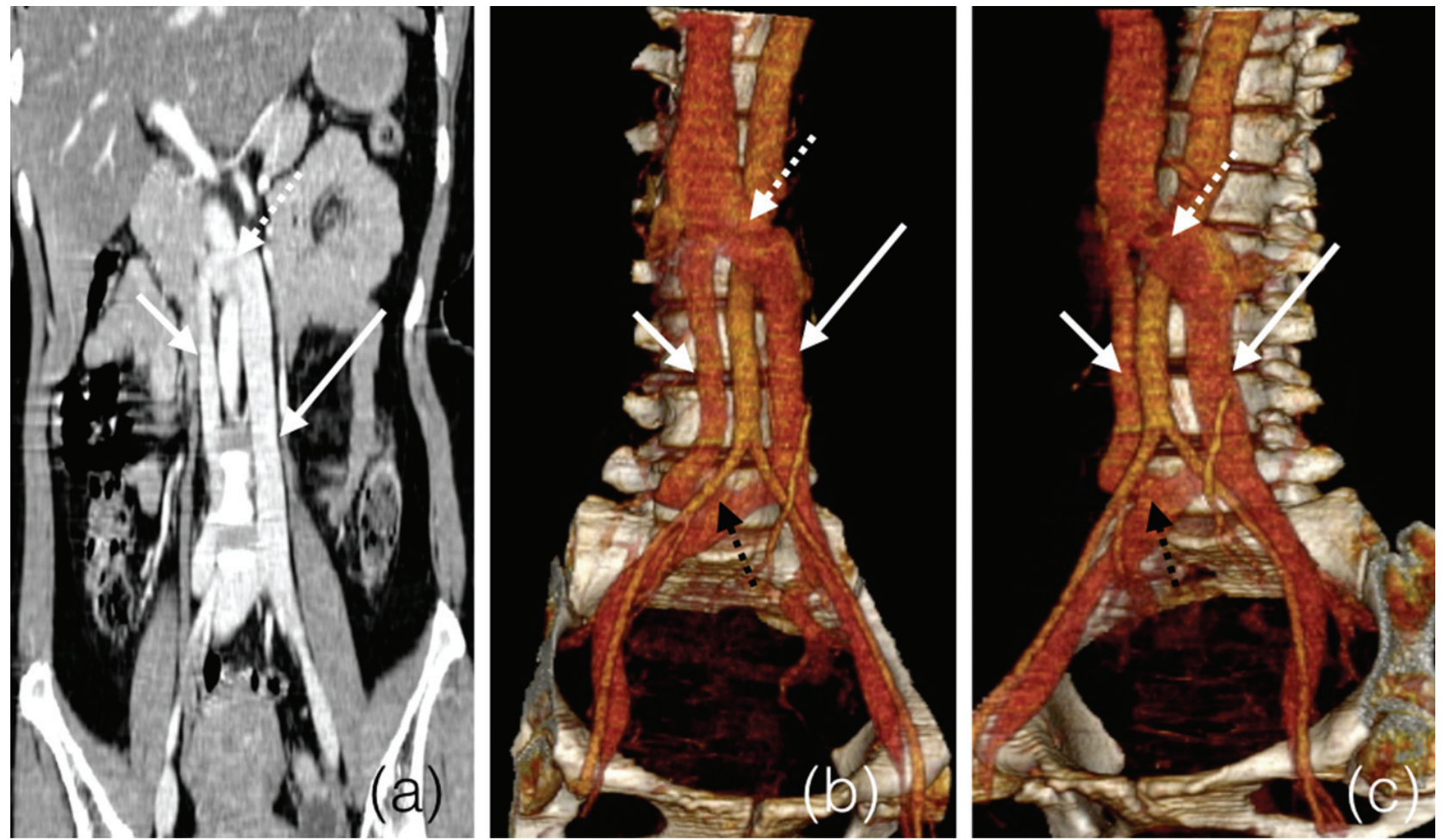

Figure 2: Coronal reconstruction and 3D Volume Rendering images: The left IVC (long arrow) crossed the midline (dashed white arrow). The right IVC (short arrow) continuous inferiorly with the right common iliac vein (dashed black arrow).

Most patients with IVC anomalies are asymptomatic ${ }^{2,3}$. As in our patients, the anomalies were identified incidentally during imaging for other medical conditions. Some authors have divided the IVC anomalies into: a) duplication of the IVC, b) transposition of the IVC, and c) azygos continuation of the IVC ${ }^{1,4}$. Among these IVC anomalies, the duplication and left-sided or transposition of IVC are the ones most commonly encountered ${ }^{5}$.

The duplication of IVC has reported prevalence of
$0.2-3.0 \%$. It resulted from the persistence of both supra cardinal veins or failure of regression of the left supra cardinal vein ${ }^{1,6 .}$ As a result, there is a persistent communication between the left common iliac vein and the left renal vein. The prevalence of the transposition of the IVC was $0.2-0.5 \%^{1,3}$. There were regression of the right supra cardinal vein and persistence of the left supra cardinal vein. In both IVC anomalies, the left renal vein crosses to the right, anterior to the abdominal aorta and unites with the normal suprarenal IVC'. 

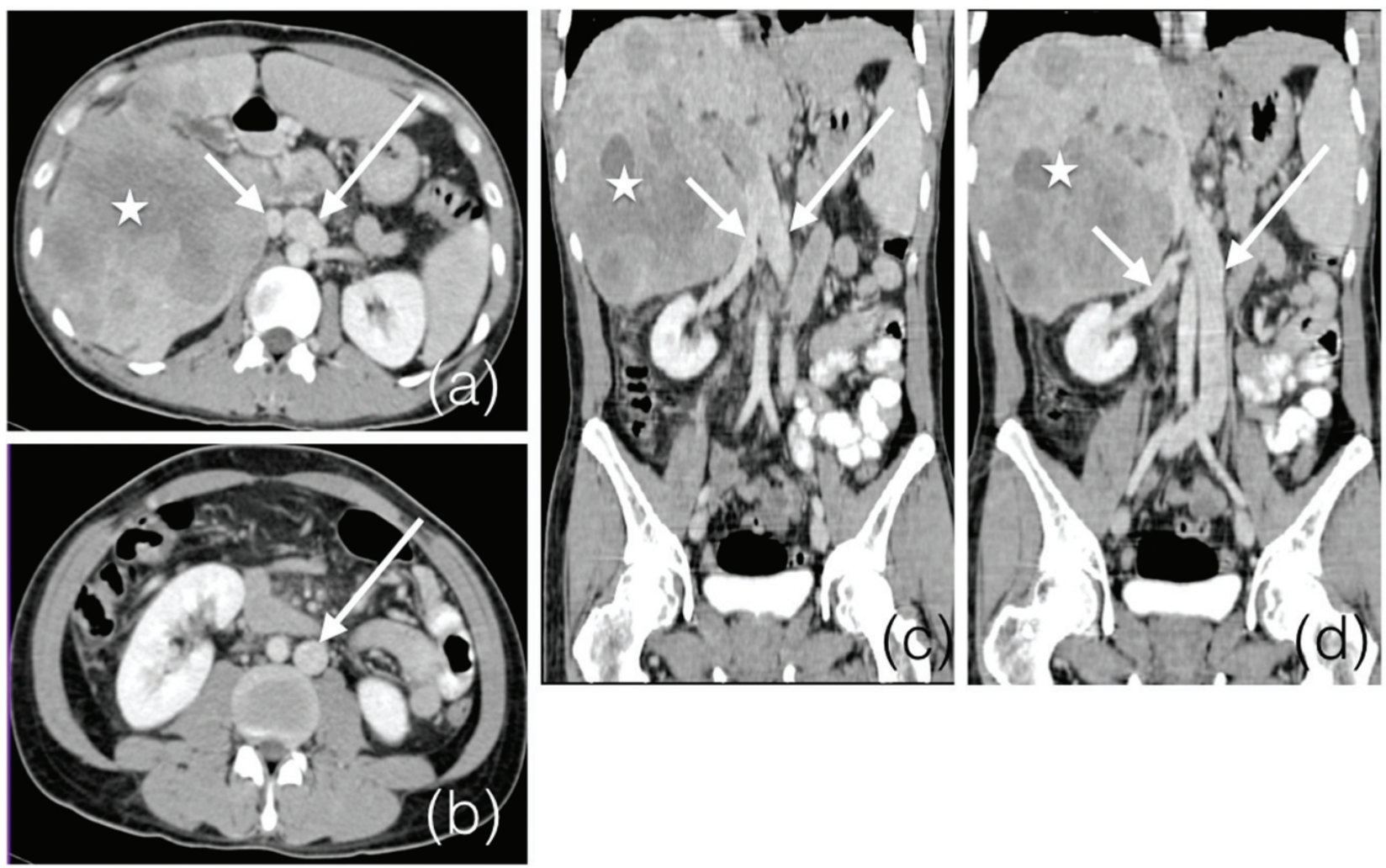

Figure 3: Axial CT (a), (b) and Coronal reconstruction (c) and (d) images. Hepatoma (asterisk) in right liver lobe. The infrarenal IVC (long arrow) continuous with the suprarenal IVC. The right renal vein (short arrow) drains into the suprarenal IVC.
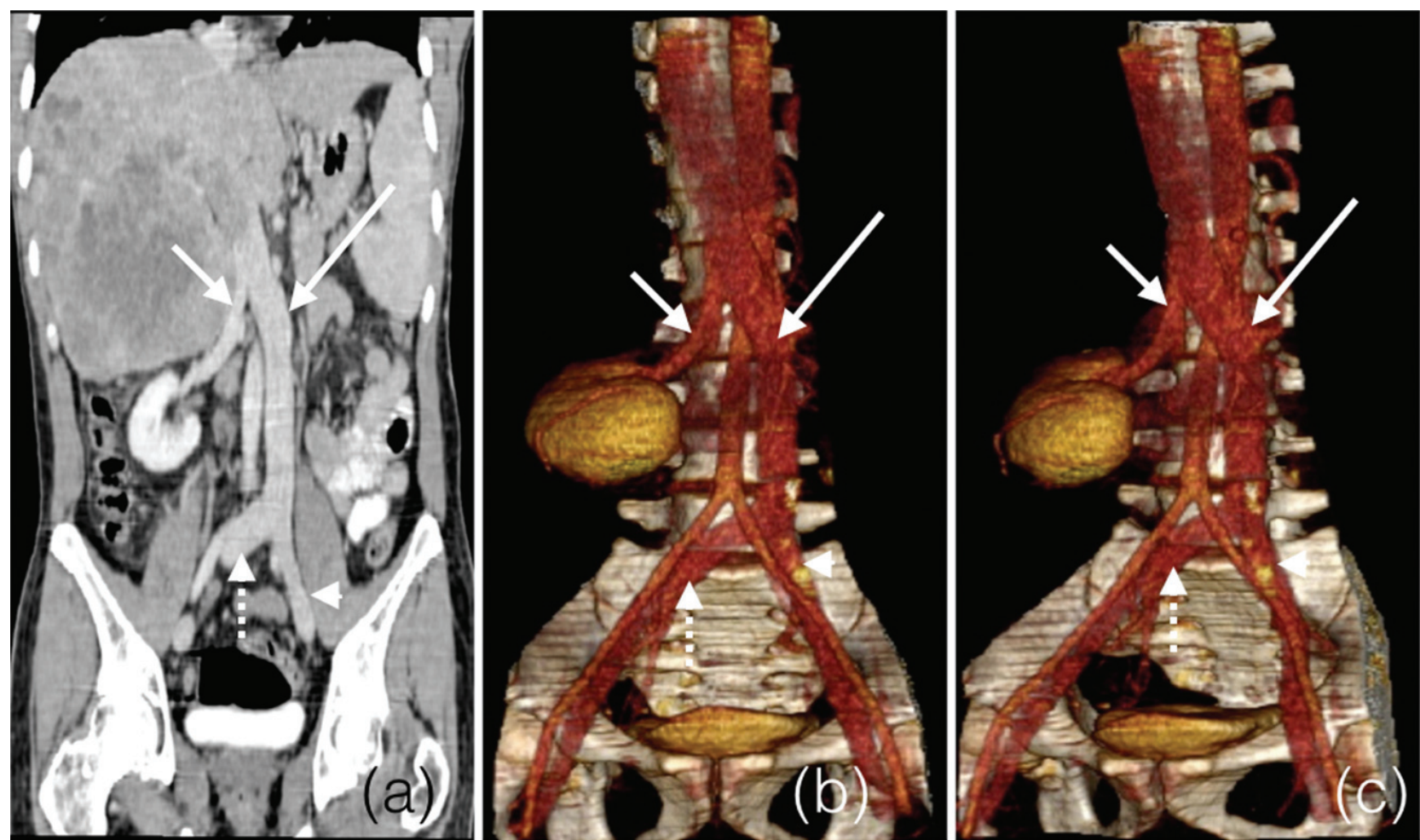

Figure 4: Coronal reconstruction and 3D Volume Rendering images. The left IVC (long arrow) received venous drainage from the right common (dashed arrow) and left common (arrow head) iliac veins. The right renal vein drains into the suprarenal IVC (short arrow). 
The awareness of the existing IVC anomalies is important to avoid catastrophic complication during abdominal procedure such as caval filtration and retroperitoneal surgeries ${ }^{7}$. On non-contrast CT, the left IVC appeared as soft tissue mass and could be misinterpreted as para aortic lymphadenopathy, ureteric dilatation, dilated left gonadal and hemiazygos vein ${ }^{8}$. The advances in the development of CT scan with the ability of reconstruction allow the detection and assessment of these vascular anomalies.

\section{CONCLUSION}

Even though IVC anomaly is rare, it is important to have knowledge about the existence and variants. With the advent of cross-sectional imaging especially $\mathrm{CT}_{\text {, the }}$ variants should be identified and reported to avoid unnecessary injuries during procedures.

\section{REFERENCES}

1. Bass JE, Redwine MD, Kramer LA, Huynh PT, Harris JH Jr. Spectrum of congenital anomaliesof theinferior vena cava: cross-sectional imaging findings. Radio Graphics. 2000 May-Jun;20(3):639-52.

2. Chen H, Emura S, Nagasaki S, Kubo KY. Double inferior vena cava with inter iliac vein: a case report and literature review. Okajimas Folia AnatJpn. 2012 Feb;88(4):147-151.

3. Luo XL, Qian GN, Xiao H, Zhao CL, Zhou XD. Posterior nutcracker syndrome associated with interrupted left inferior vena cava with azygos continuation and retroaortic right renal vein. Korean J Radiol. 2012 Jun;13(3):345-349.
4. Ng WT, Ng SS. Double inferior vena cava: a report of three cases. Singapore Med J. 2009 Jun;50(6):211213.

5. Gupta P, Khullar M, Sharma R, Singh R. A Rare Presentation of the Double Inferior Vena Cava with an Anomalous Retrocaval Right Ureter: Embryogenesis and Clinical Implications. J ClinDiagn Res. 2013 Mar;7(3):518-521.

6. Amezyane T, Bassou D, Ghafir D, Ohayon V. Double inferior vena cava mimicking lymphadenopathy. Ann Saudi Med. 2011 Nov-Dec;31(6):663.

7. ZanganSM, Van Ha TG. Duplicationof thelnferior Vena Cava. ApplRadiol. 2012;41(5):30A-30B.

8. Evans JC, Earis J, Curtis J.Thrombosed double inferior vena cavamimicking paraaortic lymphadenopathy. Br J Radiol 2001 Feb;74(878):192-194. 